\section{Learning from others: The new global conservation planning database}

Creating a new marine management or conservation plan? You can learn what others have done in the past - build on their research and experiences and avoid making the same mistakes - using the new Conservation Planning Database. The database has just been launched with 163 peer-reviewed papers on 155 marine systematic conservation planning exercises worldwide. The database can help planners find relevant conservation plans from all over the world including their local area, help scientists study trends in conservation planning, and help donors and NGOs identify regions where little conservation planning has been done.

\section{Learning from the database}

According to lead database author Jorge Álvarez-Romero, a research fellow at the ARC Centre of Excellence for Coral Reef Studies at James Cook University, pulling the initial database together was difficult, even with its focus on just peer-reviewed literature. This difficulty was due in large part to the recent surge in systematic conservation planning (and its attendant literature) worldwide. The rapid expansion of the literature means, however, that the time is ripe for a conservation planning database because there is tremendous potential for analyzing and synthesizing systematic conservation planning work and learning from it.

And, indeed, a new publication in Biological Conservation took an initial look at the systematic conservation plans in the database and found a number of trends including:

- Increasing consideration of socioeconomic variables, land-sea planning, ecological connectivity, and climate change in conservation planning studies;

- Limited involvement of stakeholders in many planning exercises;

- A concentration of studies in the Northern European Seas, Mediterranean Sea, Coral Triangle Tropical Southwestern Pacific, Temperate Northern Pacific and off the coasts of South Africa, Australia, Chile, and the US; and

- Regions with high levels of anthropogenic impact but very few conservation planning studies, e.g. the South European Atlantic Shelf, Saharan Upwelling, Azores Canaries Madeira ecoregion, West and South Indian shelf, South China Sea, and Caribbean.

\section{What's next for the database?}

James Cook University and partners, including the UNEP-World Conservation Monitoring Centre (WCMC), Imperial College, and the University of Maine, are now working to expand and improve the database. A top priority is adding a GIS/spatial module to allow storage of planning boundaries and maps of conservation priorities. "This information is practically absent from the peer reviewed literature and very patchy in the grey literature," says Álvarez-Romero. "Having this information will allow very powerful visualization and spatial analyses that are not possible yet," he adds.

Other critical next steps for the database include:
This is a summary of: Research advances and gaps in marine planning: towards a global database in systematic conservation planning

Accessible at: https://marxiv.org/h2jfv

Authors: Jorge ÁlvarezRomero, Morena Mills, Vanessa Adams, Georgina Gurney, Robert Pressey, Rebecca Weeks, Natalie Ban, Jessica Cheok, Tammy Davies, Jon Day, Mélanie Hamel, Heather Leslie, Rafael Magris, and Collin Storlie

Added to MarXiv:

September 2018

Published: Biological Conservation, 2018

Suggested Citation: Learning from others: The new global conservation planning database. OCTO (2018). DOI: 10.31230/osf. io/zq5aw

See more MarXiv summaries at https://www.marxivinfo.org/ summaries

Share your research in MarXiv and the MarXiv Team may summarize it, just like this!

Step-by-step instructions on how to share your research in MarXiv are detailed at https://www.marxivinfo.org/ submission

Across all disciplines, papers available for free with a preprint (also known as "Green OA") are cited $30 \%$ more than the world average (DOI: 10.7287/peerj. preprints.3119v1) 


\section{MarXiv Summary}

MarXiv: The free research repository for the ocean \& marine-climate sciences

- Confirming that all of the marine systematic conservation planning work from the primary literature is in the database;

- $\quad$ Adding the grey literature to the database;

- Adding terrestrial and freshwater plans;

- Adding information about implementation and monitoring of conservation interventions associated with plans;

- Linking to other global databases, such as the World Database of Protected Areas, to track the development, implementation and impact of conservation planning; and

- Planning for the long-term hosting and maintenance of the database.

\section{Add your own conservation planning exercises to the database}

Planning exercises that: 1) define explicit conservation objectives, 2) identify spatially-explicit conservation areas, 3) use spatial optimization/prioritization, and 4) identify marine conservation areas are appropriate for addition to the database.

Use this online survey to walk you through the process of adding studies.

The MarXiv Team

Nick Wehner,

Director of Open Initiatives,

OCTO

Raye Evrard,

Project Manager, ОСто

Allie Brown,

Project Associate, ОСTO

Sarah Carr,

Chief Knowledge Broker,

OCTO

John Davis,

President, OCTO

Correspondence

Nick Wehner

nick@octogroup.org

Financial support for MarXiv comes from the David and Lucile Packard Foundation.

The views expressed herein are those of the MarXiv Team and should not be interpreted as representing the opinions or policies of the Packard Foundation or other funders of MarXiv.

Join the MarXiv Summaries monthly newsletter at https:// oct.to/marxivsum

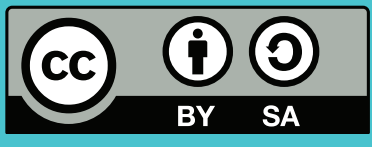

MarXiv is an ОСТO Initiative
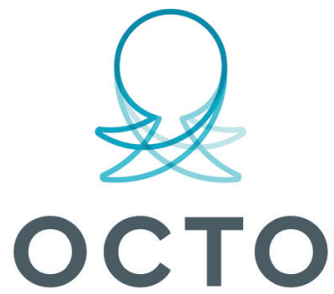

OPEN COMMUNICATIONS FOR THE OCEAN 